\title{
Expansion Mapping Theorem for Four Mappings Satisfying Common Limit in the Range Property in b-Metric Space
}

\author{
Anushree A.Aserkar ${ }^{a}$, Manjusha P.Gandhi ${ }^{b}$ \\ a Department of Applied Mathematics \& Humanities, Yeshwantrao Chavan College of Engineering, Nagpur/India. (ORCID: \\ 0000-0003-3830-2875) \\ ${ }^{\mathbf{b}}$ Department of Applied Mathematics \& Humanities, Yeshwantrao Chavan College of Engineering, Nagpur/India. (ORCID: \\ 0000-0003-1421-7701)
}

Article History: Received: 10 November 2020; Revised 12 January 2021 Accepted: 27 January 2021; Published online: 5 April 2021

\begin{abstract}
In the present paper, for four weakly compatible mappings in pairs, an expansion mapping theorem has been developed in b-metric space, meeting common limit range property. We proved this theorem without using the b-metric space's completeness condition. The result is an extension and generalization of several metric space results available. To confirm the finding, a suitable example is also discussed.
\end{abstract}

Keywords: b-metric space, expansion mapping, CLR property, weakly compatible.

\section{Introduction and Preliminary}

Banach proved a famous fixed point theorem in 1922 that ensures the existence and uniqueness of a fixed point under suitable conditions. This Banach result is known as the fixed point theorem of Banach or the theory of Banach contraction. In various ways, several writers have expanded, generalised and strengthened the fixed point theorem of Banach.

The concept of b-metric space was proposed by (Bakhtin, 1989), which was further described by (Czerwik, 1993), with a view of generalizing the Banach contraction.

1.1-b-metric space [(Bakhtin 1989), (Czerwik, 1993)]: Let $X$ be a (nonempty) set and $s \geq 1$ be a given real number. A function $d: X \times X \rightarrow[0, \infty)$ is a b-metric on $X$ if the following conditions hold:

(i) $d(x, y)=0$ if and only if $x=y$

(ii) $d(x, y)=d(y, x)$ for any $x, y \in X$

(iii) $d(x, y) \leq s\{d(x, z)+d(z, y)\}$ for any $x, y, z \in X$

Then the pair $(X, d)$ is called a b-metric space (or metric type space).

Some interesting work on expansion mappings in metric spaces was proposed by (Wang et al. 1984).

1.2-Theorem [(Wang et al., 1984)]: Let $A$ is a self-map of a complete metric space $(X, d)$. If there exist constant $a>1$ such that $d(A x, A y) \geq a d(x, y)$ for all $x, y \in X$ and $A$ is onto, then $A$ has a unique fixed point.

1.3-Theorem [(Wang et al., 1984)]: Let $A$ is a self-map of a complete metric space $(X, d)$. If there exists non-negative constants $a, b, c$ such that $a+b+c>1$ and $a<1$ such that $d(A x, A y) \geq a d(x, y)+b d(A x, x)+c d(A y, y)$ for all $x, y \in X, x \neq y$ and $A$ is onto, then $A$ has a unique fixed point.

For multiple mappings, numerous researchers have proved fixed point theorems. In some instances, to obtain a common fixed point, commutative properties between the maps are required. The term Weak Commutative Mappings was initiated by (Sessa, 1982). By introducing the concept of compatible maps, (Jungck, 1986) extended the notion of weak commutative state, and then in (Jungck, 1996)introduced weakly compatible maps.

1.4-Weakly compatible map [(Jungck, 1996)]: Let two self-maps $A$ and $B$ be defined on a set $X$, then $A$ and $B$ are said to be weakly compatible if they commute at points of coincidence. That is if $A x=B x$ for some $x \in X$, then $A B x=B A x$..

The concept of E.A. was put forward by (Aamri and Moutawakil, 2002), which is a true generalisation of non-compatible maps in metric spaces. (Sintunavarat and Kumam, 2011) launched the concept of common limit 
in the range (CLR) property for a pair of self-mappings in Fuzzy metric space. This property is outstanding since it does not need the closed subspaces.

1.5-Common limit in the range property [(Sintunavarat and Kumam, 2011)]: Suppose that $(X, d)$ is a metric space and $A, B: X \rightarrow X$. Two mappings $A$ and $B$ are said to satisfy the common limit in the range of $B$ property if $\lim _{n \rightarrow \infty} A x_{n}=\lim _{n \rightarrow \infty} B x_{n}=B x$ for some $x \in X$

The property was extended by (Wu et.al., 2017) to b-metric space for three mappings.

Let $A, B, T: X \rightarrow X$ be three self-mappings of a b-metric space $(X, d)$. The pair $A, B$ satisfies the common limit in the range of $T$ property $\left(C L R_{T}\right)$ if there exists a sequence $\left\{x_{n}\right\} \subseteq X$ and a point $x \in X$ such that $\lim _{n \rightarrow \infty} A x_{n}=\lim _{n \rightarrow \infty} B x_{n}=T x \in T X$.

Specifically, if $B=T$ the pair $A, B$ satisfies the $\left(C L R_{B}\right)$-property.

The result-1.2 was expanded in (Daffer and Kaneko, 1993) for two mappings. For two compatible mappings, (Rhoades, 1993) generalized the result-1.2.

(Manro and Kumar, 2012) demonstrated the result-1.2 for two mappings that satisfy either compatible or Rweak type commuting ( $\mathrm{Ag}$ ) or R-weak type commuting (Af) or R-weak type commuting (Af) or R-weak type commuting $(\mathrm{P})$. The result-1.2 for partially ordered metric space has been extended by (Huang et.al. 2012) for two mappings satisfying weakly compatible conditions in pairs.

For b-metric space with four weakly compatible mappings in pairs that satisfy the CLR property in pairs, the key result in this paper is proven. All four subsets are not closed. All the above results (Wang et al., 1984, Daffer and Kaneko, 1993, Rhoades, 1993, Manro and Kumar, 2012, Huang et.al. 2012) are extended and generalized by our outcome.

\section{Main Result}

For four weakly compatible mappings in pairs that satisfy common limit range properties, an expansion mapping theorem is defined in b-metric space.

2.1-Theorem: Let $(X, d)$ be a b-metric space with $s \geq 1$ and $A, B, S, T: X \rightarrow X$ be mappings with $A(X) \subseteq T(X)$ and $B(X) \subseteq S(X)$ such that

(i) $d(T x, S y) \geq a \min \{d(A x, B y), d(S y, B y), d(A x, T x)\}+\frac{b}{s}\left\{\frac{d(A x, S y)+d(T x, B y)}{2}\right\}$

for all $x, y \in X, a \geq 1, b \geq 0, \frac{b}{s}<1$.

(ii) $A, T$ satisfies $C L R_{S}$ and $B, S$ satisfies $C L R_{T}$ property.

(iii) The pairs $(A, T)$ and $(B, S)$ are weakly compatible.

Then $A, B, S, T$ have a unique common fixed point.

Proof: As $A, T$ satisfies $C L R_{S}$ and $B, S$ satisfies $C L R_{T}$ property, there exists $\left\{x_{n}\right\}$ and $\left\{y_{n}\right\}$ in $X$ and $\alpha, \beta \in X$ such that $\lim _{n \rightarrow \infty} A x_{n}=\lim _{n \rightarrow \infty} T x_{n}=S \alpha \in S(X)$ and $\lim _{n \rightarrow \infty} B y_{n}=\lim _{n \rightarrow \infty} S y_{n}=T \beta \in T(X)$.

Putting $x=x_{n}$ and $y=y_{n}$ in (2.1.1),

$$
d\left(T x_{n}, S y_{n}\right) \geq a \min \left\{d\left(A x_{n}, B y_{n}\right), d\left(S y_{n}, B y_{n}\right), d\left(A x_{n}, T x_{n}\right)\right\}+\frac{b}{s}\left\{\frac{d\left(A x_{n}, S y_{n}\right)+d\left(T x_{n}, B y_{n}\right)}{2}\right\}
$$

Taking $\lim _{n \rightarrow \infty}$ to both sides, we get

$$
\begin{aligned}
\lim _{n \rightarrow \infty} d\left(T x_{n}, S y_{n}\right) \geq a \lim _{n \rightarrow \infty} \min \left\{d\left(A x_{n}, B y_{n}\right),\right. & \left.d\left(S y_{n}, B y_{n}\right), d\left(A x_{n}, T x_{n}\right)\right\} \\
& +\lim _{n \rightarrow \infty} \frac{b}{s}\left\{\frac{d\left(A x_{n}, S y_{n}\right)+d\left(T x_{n}, B y_{n}\right)}{2}\right\}
\end{aligned}
$$




$$
\begin{array}{r}
d(S \alpha, T \beta) \geq a \min \{d(S \alpha, T \beta), d(T \beta, T \beta), d(S \alpha, S \alpha)\}+\frac{b}{s}\left\{\frac{d(S \alpha, T \beta)+d(S \alpha, T \beta)}{2}\right\} \\
\therefore d(S \alpha, T \beta) \geq \frac{b}{s} d(S \alpha, T \beta)
\end{array}
$$$$
\therefore\left(\frac{b}{s}-1\right) d(S \alpha, T \beta) \leq 0 \Rightarrow d(S \alpha, T \beta)=0 \quad \mathrm{Q} \frac{b}{s}<1 \Rightarrow \frac{b}{s}-1 \neq 0
$$$$
\therefore S \alpha=T \beta
$$

Putting $x=x_{n}$ and $y=\alpha$ in (2.1.1),

$d\left(T x_{n}, S \alpha\right) \geq a \min \left\{d\left(A x_{n}, B \alpha\right), d(S \alpha, B \alpha), d\left(A x_{n}, T x_{n}\right)\right\}+\frac{b}{s}\left\{\frac{d\left(A x_{n}, S \alpha\right)+d\left(T x_{n}, B \alpha\right)}{2}\right\}$

Taking $\lim _{n \rightarrow \infty}$ to both sides, we get

$$
\begin{aligned}
& \lim _{n \rightarrow \infty} d\left(T x_{n}, S \alpha\right) \geq a \lim _{n \rightarrow \infty} \min \left\{d\left(A x_{n}, B \alpha\right), d(S \alpha, B \alpha), d\left(A x_{n}, T x_{n}\right)\right\} \\
& \quad+\frac{b}{s} \lim _{n \rightarrow \infty}\left\{\frac{d\left(A x_{n}, S \alpha\right)+d\left(T x_{n}, B \alpha\right)}{2}\right\} \\
& d(S \alpha, S \alpha) \geq a \min \{d(S \alpha, B \alpha), d(S \alpha, B \alpha), d(S \alpha, S \alpha)\}+\frac{b}{s}\left\{\frac{d(S \alpha, S \alpha)+d(S \alpha, B \alpha)}{2}\right\} \\
& \therefore\left(\frac{b}{2 s}\right) d(S \alpha, B \alpha) \leq 0 \quad \mathrm{Q} b \geq 0 \Rightarrow \frac{b}{2 s} \geq 0 \\
& d(S \alpha, B \alpha)=0 \Rightarrow S \alpha=B \alpha
\end{aligned}
$$

$\therefore$ from (2.1.2), (2.1.3), (2.1.4) we get

$$
A \beta=T \beta=S \alpha=B \alpha
$$

Let $A \beta=T \beta=S \alpha=B \alpha=\gamma$

$\mathrm{Q} A, T$ are weakly compatible,

$A \gamma=A T \beta=T A \beta=T \gamma$

Putting $x=\gamma, y=\alpha$ in (2.1.1), we get

$$
\begin{aligned}
& d(T \gamma, S \alpha) \geq a \min \{d(A \gamma, B \alpha), d(S \alpha, B \alpha), d(A \gamma, T \gamma)\}+\frac{b}{s}\left\{\frac{d(A \gamma, S \alpha)+d(T \gamma, B \alpha)}{2}\right\} \\
& \therefore d(T \gamma, \gamma) \geq a \min \{d(T \gamma, \gamma), d(\gamma, \gamma), d(T \gamma, T \gamma)\}+\frac{b}{s}\left\{\frac{d(T \gamma, \gamma)+d(T \gamma, \gamma)}{2}\right\} \\
& \therefore d(T \gamma, \gamma) \geq \frac{b}{s} d(T \gamma, \gamma) \\
& \left(\frac{b}{s}-1\right) d(T \gamma, \gamma) \leq 0 \Rightarrow d(T \gamma, \gamma)=0 \quad \mathrm{Q} \frac{b}{s}<1 \Rightarrow \frac{b}{s}-1 \neq 0 \\
& \therefore T \gamma=\gamma \\
& \therefore A \gamma=T \gamma=\gamma
\end{aligned}
$$

Similarly we may prove that $S \gamma=B \gamma=\gamma$

$\therefore A \gamma=B \gamma=S \gamma=T \gamma=\gamma$

Uniqueness: Let if possible there are two fixed points $\gamma, \gamma^{*}$ i.e. $A \gamma=B \gamma=S \gamma=T \gamma=\gamma$ and $A \gamma^{*}=B \gamma^{*}=S \gamma^{*}=T \gamma^{*}=\gamma^{*}$.

Putting $x=\gamma, y=\gamma^{*}$ in (2.1.1), we get 


$$
\begin{aligned}
& d\left(T \gamma, S \gamma^{*}\right) \geq a \min \left\{d\left(A \gamma, B \gamma^{*}\right), d\left(S \gamma^{*}, B \gamma^{*}\right), d(A \gamma, T \gamma)\right\}+\frac{b}{s}\left\{\frac{d\left(A \gamma, S \gamma^{*}\right)+d\left(T \gamma, B \gamma^{*}\right)}{2}\right\} \\
& d\left(\gamma, \gamma^{*}\right) \geq a \min \left\{d\left(\gamma, \gamma^{*}\right), d\left(\gamma^{*}, \gamma^{*}\right), d(\gamma, \gamma)\right\}+\frac{b}{s}\left\{\frac{d\left(\gamma, \gamma^{*}\right)+d\left(\gamma, \gamma^{*}\right)}{2}\right\} \\
& \therefore d\left(\gamma, \gamma^{*}\right) \geq \frac{b}{s} d\left(\gamma, \gamma^{*}\right) \\
& \therefore\left(\frac{b}{s}-1\right) d\left(\gamma, \gamma^{*}\right) \leq 0 \quad \mathrm{Q} \frac{b}{s}<1 \Rightarrow \frac{b}{s}-1 \neq 0 \\
& \therefore \gamma=\gamma^{*}
\end{aligned}
$$

Thus the fixed point is unique.

2.2-Corollary: Let $(X, d)$ be a metric space and $A, B, S, T: X \rightarrow X$ be mappings with $A(X) \subseteq T(X)$ and $B(X) \subseteq S(X)$ such that

(i) $d(T x, S y) \geq a \min \{d(A x, B y), d(S y, B y), d(A x, T x)\}$ for all $x, y \in X, a \geq 1$.

(ii) $A, T$ satisfies $C L R_{S}$ and $B, S$ satisfies $C L R_{T}$ property.

(iii) The pairs $(A, T)$ and $(B, S)$ are weakly compatible.

Then $A, B, S, T$ have a unique common fixed point.

Proof: If we substitute $b=0$ in theorem-2.1, the result is obtained. The result is a generalization of the results (Wang et al., 1984, Daffer and Kaneko, 1993, Rhoades, 1993, Manro and Kumar, 2012, Huang et.al. 2012) with four mappings satisfying CLR property which is more recent and generalized compatibility condition.

2.3-Corollary: Let $(X, d)$ be a b-metric space with $s \geq 1$ and $A, B, S, T: X \rightarrow X$ be mappings with $A(X) \subseteq T(X)$ and $B(X) \subseteq S(X)$ such that

(i) $d(T x, S y) \geq a \min \left\{d(A x, B y), d(S y, B y), d(A x, T x), \frac{d(A x, S y)+d(T x, B y)}{2 s}\right\}$

for all $x, y \in X, a \geq 1$.

(ii) $A, T$ satisfies $C L R_{S}$ and $B, S$ satisfies $C L R_{T}$ property.

(iii) The pairs $(A, T)$ and $(B, S)$ are weakly compatible.

Then $A, B, S, T$ have a unique common fixed point.

Proof: If we substitute $a=b$ in theorem-2.11,

$$
\begin{gathered}
d(T x, S y) \geq a \min \{d(A x, B y), d(S y, B y), d(A x, T x)\}+a\left\{\frac{d(A x, S y)+d(T x, B y)}{2 s}\right\} \\
\geq a \min \left\{d(A x, B y), d(S y, B y), d(A x, T x), \frac{d(A x, S y)+d(T x, B y)}{2 s}\right\}
\end{gathered} .
$$

2.4-Example: Let $X=[1,6]$ and $d: X \times X \rightarrow[0, \infty)$ be defined by $d(x, y)=|x-y|$. We define mappings

$$
A x=\left\{\begin{array}{ll}
3, & x \leq 3, \\
4, & x>3 .
\end{array} \quad B x=\left\{\begin{array}{ll}
4, & x<3, \\
\frac{x+3}{2}, & x \geq 3 .
\end{array} \quad T x=\left\{\begin{array}{ll}
6-x, & x \leq 3, \\
5, & x>3 .
\end{array} \quad \text { and } S x= \begin{cases}6, \quad x<3, \\
\frac{2 x+3}{3}, & x \geq 3 .\end{cases}\right.\right.\right.
$$

Obviously, with $\mathrm{s}=2, \mathrm{~d}$ is a b-metric.

To prove that $A, T$ satisfies $C L R_{S}$ and $B, S$ satisfies $C L R_{T}$ property, consider sequences $\left\{x_{n}\right\}$ and $\left\{y_{n}\right\}$ defined by $x_{n}=3-\frac{1}{n}$ and $y_{n}=3+\frac{1}{n}$.

We have $\lim _{n \rightarrow \infty} A x_{n}=\lim _{n \rightarrow \infty} T x_{n}=3=S 3 \in S X$ and $\lim _{n \rightarrow \infty} B y_{n}=\lim _{n \rightarrow \infty} S y_{n}=3=T 3 \in T X$ 
It is easily proved that $A, T$ and $B, S$ are weakly compatible.

Clearly $A(X)=\{3,4\} \subseteq[3,5]=T(X), B(X)=[3,4.5] \subseteq[3,5]=S(X)$

$a=\frac{6}{5}, b=1, s=2$

Case-1: $\mathrm{x}<3, \mathrm{y}<3 \quad$ Let $x=2$

$A x=3, \quad B x=4, \quad T x=6-x, \quad S x=6$

$L H S=d(T x, S y)=|6-x-6|=2$

$R H S=a \min \{d(A x, B y), d(S y, B y), d(A x, T x)\}+\frac{b}{s}\left\{\frac{d(A x, S y)+d(T x, B y)}{2}\right\}$

$=\frac{6}{5} \min \{|3-4|,|6-4|,|6-2-3|\}+\frac{1}{2}\left\{\frac{|3-6|+|6-2-4|}{2}\right\}$

$=\frac{6}{5}+\frac{3}{4}=\frac{39}{20}$

$\therefore L H S \geq R H S$

Case-II: $\mathrm{x}=3, \mathrm{y}=3$

$A x=3, \quad B x=3, \quad T x=3, \quad S x=3$

$L H S=d(T x, S y)=|3-3|=0$

$R H S=a \min \{d(A x, B y), d(S y, B y), d(A x, T x)\}+\frac{b}{s}\left\{\frac{d(A x, S y)+d(T x, B y)}{2}\right\}=0$

$\therefore$ LHS $\geq R H S$

Case-III: $\mathrm{x}>3, \mathrm{y}>3$

$A x=4, \quad B y=\frac{y+3}{2}, \quad T x=5, \quad S y=\frac{2 y+3}{3} \quad$ Let $y=4$

$L H S=d(T x, S y)=\left|5-\frac{11}{3}\right|=\frac{4}{3}$

$R H S=a \min \{d(A x, B y), d(S y, B y), d(A x, T x)\}+\frac{b}{s}\left\{\frac{d(A x, S y)+d(T x, B y)}{2}\right\}$

$=\frac{6}{5} \min \left\{|4-3.5|,\left|\frac{11}{3}-\frac{7}{2}\right|,|5-4|\right\}+\frac{1}{2}\left\{\frac{|4-11 / 3|+|5-3.5|}{2}\right\}$

$=\frac{6}{5} \min \left\{\frac{1}{2}, \frac{1}{6}, 1\right\}+\frac{1}{2} \cdot \frac{11}{12}=\frac{1}{5}+\frac{11}{24}=\frac{79}{120}$

$\therefore L H S \geq R H S$

Thus all the conditions of the theorem is satisfied. Hence the fixed point is at $x=3$.

We may check that $A 3=B 3=T 3=S 3=3$.

\section{Acknowledgements}

The authors are thankful to the affiliated college authorities for the motivation given by them.

\section{References}

[1]. Bakhtin, I.A.(1989). The contraction principle in quasimetric spaces, Funct. Analysis, 30, $26-37$.

[2]. Czerwik, S. (1993). Contraction mappings in b-metric spaces, Acta Math. Inform., Univ. Ostrav.,1,5-11.

[3]. Wang, S.Z. , Li, B.Y., Gao, Z.M., Iseki, K. (1984), Some Fixed Point Theorems On Expansion Mappings. Math. Jpn. 29. 
[4]. Sessa, S. (1982). On a weak commutativity condition of mappings in fixed point consideration. Publ. Inst. Math. 32, 149-153.

[5]. Jungck, G. (1986). Compatible mappings and common fixed points. . Int. J. Math. Math. Sci. 9, 771779.

[6]. Jungck, G. (1996). Common fixed points for non-continuous non-self maps on non-metric spaces, Far East J. Math. Sci. 4 (2), 199-215.

[7]. Aamri, M. Moutawakil, Del. (2002). Some new common fixed point theorems under strict contractive

[8]. conditions. J. Math. Anal. Appl. 270, 181-188.

[9]. Sintunavarat, W. Kumam, P. (2011). Common fixed point theorems for a pair of weakly compatible

[10]. mappings in fuzzy metric spaces, Journal of Applied Mathematics, Article ID 637958,14 pages.

[11]. Wu, B. Fei, He. Tao, Xu. (2017). Common fixed point theorems for Ciric type mappings in b-metric spaces without any completeness assumption, J. Nonlinear Sci. Appl., 10, 3180-3190.

[12]. Daffer, Z.P. Kaneko, H.(1992). On Expansive Mappings, Math. Japonica, 7, 733-735.

[13]. Rhoades, B.E. (1993). An expansion mapping theorem, Jnanabha, 23, 151-152.

[14]. Manro, S. Kumam, P. (2012). Common fixed point theorems for expansion mappings in various abstract spaces using the concept of weak reciprocal continuity, http://www.fixedpointtheoryandapplications.com/content/2012/1/221

[15]. Huang, X. Zhu, C. and Wen, Xi. (2012). Fixed point theorems for expanding mappings in cone metric spaces, math. Reports, 14(64), 141-148. 